\title{
Computational Rock Physics Using a Hamiltonian Particle Method for Effective Elastic Properties of Cracked Media
}

\author{
Junichi TAKEKAWA ${ }^{1}$, Hitoshi MIKADA ${ }^{1}$ and Tada-nori GOTO ${ }^{1}$ \\ ${ }^{1}$ Dept. of Civil and Earth Res. Eng., Kyoto University
}

\begin{abstract}
It is important to estimate physical properties of cracked rocks in exploration geophysics. In the past, many analytical studies for elastic properties of a cracked medium have been conducted, for example non-interacting theory, self-consistent theory and so on. However, some limitations (e.g. crack geometries, long wavelength limit) always remain in theoretical approaches. On the other hand, numerical studies, which are much less restrictive, have also been conducted using several numerical methods. In the present study, we apply a Hamiltonian particle method (HPM) for estimating effective elastic properties of cracked media because HPM has some advantages in treating cracked media. In order to investigate the validity of the particle method for determining the effective properties of cracked media, we calculate the effective velocity of cracked region using the particle method and compare with those from several effective medium theories. Our results show good agreements with those from modified self-consistent theory. Since this is consistent with the previous studies, HPM can estimate effective elastic properties of cracked media. HPM has some advantages about computational costs, this indicates that the effective calculation with low computation costs (numerical memory and calculation costs) is possible.
\end{abstract}

\section{INTRODUCTION}

The prediction of effective elastic properties of cracked solids is of considerable interest for geophysics, civil and environmental engineering. In the past, many analytical studies for elastic properties of a cracked medium have been conducted. Davis and Knopoff ${ }^{1)}$ showed that the mean-field approximation is appropriate for the problem of the modulus of a high concentration of cracks by using a boundary integral method. Henyey and Pomphrey $^{2)}$ proposed modified self-consistent theory with a set of differential equations for the effective elastic moduli taking into account the crack interaction energy. Although many researchers have proposed a variety of approaches, some limitations (e.g. crack geometries, long wavelength limit) always remain in theoretical approaches. On the other hand, numerical studies, which are much less restrictive, have also been conducted using several numerical methods. Saenger and Shapiro ${ }^{3)}$ conducted finite difference simulations with rotated staggered grid ${ }^{4)}$ in order to investigate effective elastic properties of fractured 2D medium. Their results were in good agreement with the predictions of the modified self-consistent theory. In finite difference method, however, it is difficult to refine the grid in space locally. Thus, models of analysis need to be discretized by fine grids whose spatial scale is adjusted for representing cracks in the models.

Recently, Takekawa et al. ${ }^{5}$ applied a Hamiltonian particle method (HPM) ${ }^{6}$ ) to simulate surface wave propagation taking advantage of a simple introduction of traction free boundary conditions. In HPM, the free surface can be introduced just by ignoring the interactions with outer media. This feature can be applicable to reproduce free-surface inside the rock masses instead of the introduction of inhomogeneities with high contracts of elastic parameters and density. Arbitrary refinement of spatial resolution is also applicable in a simple and easy manner using $\mathrm{HPM}^{7}$. In Takekawa et al. ${ }^{7)}$, they refined the spatial resolution only around low velocity zone, and the numerical dispersion was suppressed in an efficient manner. This technique is also useful in refining the resolution only around crack. As described above, HPM has potential to estimate the elastic properties of cracked media.

In order to investigate the validity of the particle method for determining the effective properties of cracked media, we calculate the effective velocity of cracked region using the particle method and compare with those from several effective medium theories.

\section{METHOD}

In HPM, each particle interacts with the 
neighboring particles which are at a certain distance from it. This domain which defines the interaction area is called the influence domain. The influence domain defines a circular area or a spherical volume in two- or three-dimensional problems, respectively. The number of neighboring particles is controlled by the radius of the influence domain. The effect of the radius on seismogram will be discussed in the following chapter.

We consider a weighting function $\mathrm{w}(\mathrm{r})$ between particles to weight their interactions as follows.

$$
\mathrm{w}(\mathrm{r})=\mathrm{r}_{\mathrm{e}} / \mathrm{r}-1\left(\mathrm{r} \leq \mathrm{r}_{\mathrm{e}}\right) \text { or } 0\left(\mathrm{r}>\mathrm{r}_{\mathrm{e}}\right)
$$

where $r$ is the distance between particles and $r_{e}$ is the averaged radius of the influence domain. The weighting value at the considered particle is infinite, and becomes zero outside the influence domain. $r_{e}$ is calculated as follows,

$$
r_{e}=\left(r_{e i}+r_{e j}\right) / 2
$$

where $r_{e i}$ and $r_{e j}$ are the influence domains of particle $i$ and $j$. The influence domain of each particle depends on their radius.

$$
\mathrm{r}_{\mathrm{ei}}=\alpha \mathrm{d}_{\mathrm{i}}
$$

$d_{i}$ is the radius of particle $i, \alpha$ is the coefficient which controls the number of neighboring particles.

In HPM, the deformation gradients at the position of particle $i$ is evaluated by minimizing the error function $\mathrm{e}_{\mathrm{i}}$,

$$
\mathrm{e}_{\mathrm{i}}=\sum_{\mathrm{j}}\left|\mathbf{F}_{\mathrm{i}} \mathbf{r}_{\mathrm{ij}}^{0}-\mathbf{r}_{\mathrm{ij}}\right|^{2} \mathrm{w}_{\mathrm{ij}}^{0}
$$

where $\mathbf{F}_{\mathrm{i}}$ is the deformation gradient tensor at the position of particle $i, \mathbf{r}_{i j}^{0}$ and $\mathbf{r}_{i j}$ are the initial and current position of particle $\mathrm{j}$ relative to particle $\mathrm{i}$, respectively, $\mathrm{w}_{\mathrm{ij}}^{0}$ is the weighting function between particle $i$ and $j$ calculated by Eq.(1). We can obtain the deformation gradient tensor by minimizing Eq.(4),

$$
\begin{gathered}
\mathbf{F}_{\mathrm{i}}=\sum_{\mathrm{j}} \mathbf{r}_{\mathrm{ij}} \otimes \mathbf{r}_{\mathrm{ij}}^{0} \mathrm{w}_{\mathrm{ij}}^{0} \mathbf{A}_{\mathrm{i}}^{-1} \\
\mathbf{A}_{\mathrm{i}}=\sum_{\mathrm{j}} \mathbf{r}_{\mathrm{ij}}^{0} \otimes \mathbf{r}_{\mathrm{ij}}^{0} \mathrm{w}_{\mathrm{ij}}^{0}
\end{gathered}
$$

By adopting a constitutive law, we can calculate stresses and the total elastic strain energy.

We adopt artificial force to avoid numerical oscillation $^{8)}$. The details of HPM can be found in references $^{5,6)}$.

\section{MODEL DESCRIPTIONS}

The free-surface can be introduced just by cutting off the connectivity across the crack in HPM. This technique is simple to implement, however, the reproducibility of the crack depends on the particle spacing. To ensure enough spatial resolution, we divide one particle, which is near the crack, into four particles like in a reference $^{7)}$. This implementation is also conducted with simple procedure.

We use numerical models as shown in Figure 1. The model has a cracked region in the center of it. The cracked region is $0.2 \mathrm{~m} \times 0.2 \mathrm{~m}$, and has randomly placed, randomly oriented, rectilinear, dry and non-intersecting cracks. We use equal length of cracks, i.e. $2 \mathrm{l}_{\mathrm{k}}=0.0061 \mathrm{~m}$. In the background region, we set $2 \mathrm{D}$ isotropic elastic media and the model has a $\mathrm{V}_{\mathrm{p}}=4000 \mathrm{~m} / \mathrm{s}, \mathrm{V}_{\mathrm{s}}=2310 \mathrm{~m} / \mathrm{s}$, and $\rho=2700 \mathrm{~kg} / \mathrm{m}^{3}$. A plane wave (P- or $\mathrm{SV}$-wave) incidents from right side of the model and goes though the cracked region. We receive the plane wave passing through the cracked region with a vertical line of receivers placed just at the left of the cracked region. The source wavelet in our numerical experiments is the first derivative of a Gaussian with a dominant frequency of $20 \mathrm{kHz}$. Therefore, the dominant wavelengths of $\mathrm{P}$ - and $\mathrm{SV}$-wave in homogeneous region are $0.2 \mathrm{~m}$ and $0.1155 \mathrm{~m}$, respectively. Therefore, our numerical conditions are good approximation to reach the long wavelength limit. The particle spacing in non-refined region is $0.001 \mathrm{~m}$ and time spacing is $0.01 \mu \mathrm{s}$. We apply periodic boundary conditions in the vertical direction.

We control the number of cracks in the cracked region using crack density $\phi$ defined as follows.

$$
\phi=\frac{1}{\mathrm{~A}} \sum_{\mathrm{k}=1}^{\mathrm{n}} \mathrm{l}_{\mathrm{k}}^{2}
$$

where A represents the area of the cracked region, $n$

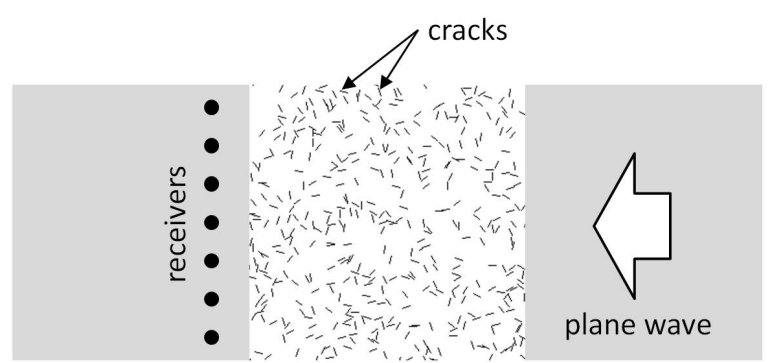

Figure 1 Schematic figure of our numerical model. Cracked region is embedded in the center of the model. 
(a)

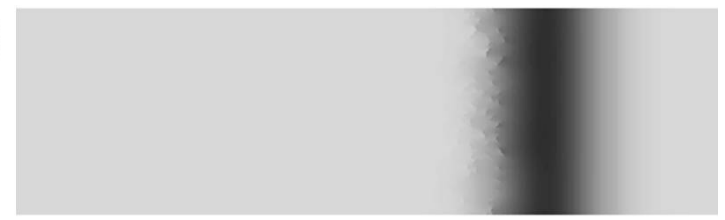

(b)

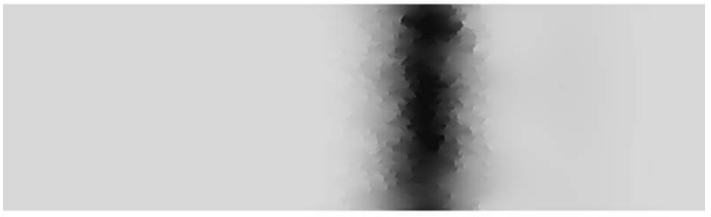

(c)

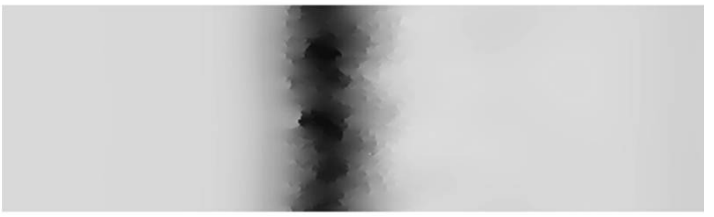

Figure 2 Snapshots of displacement of plane P-wave propagation in the cracked region. (a) after $0.135 \mathrm{~ms}$, (b) after 0.165 $\mathrm{ms}$, (c) after $0.195 \mathrm{~ms}$.

the number of cracks, $1_{k}$ the half length of each crack. If crack density increased, the number of cracks also increases.

\section{RESULTS}

Figure 2 shows snapshots of displacement for plane P-wave propagation in the cracked region. Plane wave, which is generated by enforced deformation at the right edge of the model, comes from the right side, and passes through the cracked region. We can observe that the plane wave is affected by many cracks. We record plane wave passing through the cracked region using an array of receivers placed at just left side of the region.

Figure 3 shows waveforms of displacement with different crack density. The time delay increases with increase in crack density. This means that the effective velocity of cracked region decreases with increase in crack density. This trend is consistent with theoretical considerations and laboratory observations $^{3), 9)}$. We can calculate the effective velocity of the cracked region using the observed time delay.

Figure 4 shows the calculated effective velocity with different crack densities. The error bars represent standard deviation for 10 model realizations. For comparison, we show the results from theoretical approaches in the same figure. Our numerical result has excellent agreement with that from modified self-consistent theory. This result is consistent with the previous studies conducted by

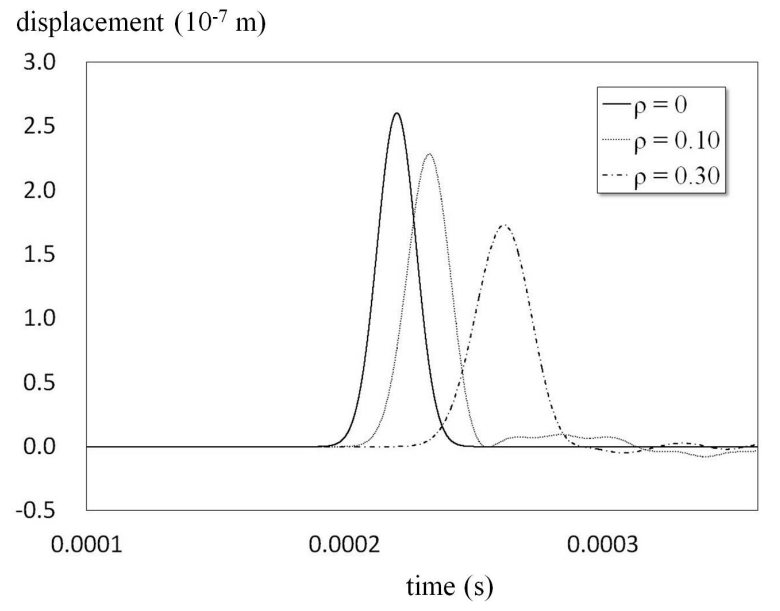

Figure 3 Displacement waveforms recorded by a receiver array. Solid, dotted and chain lines represent waveforms with crack density of $0,0.1$ and 0.3 , respectively.

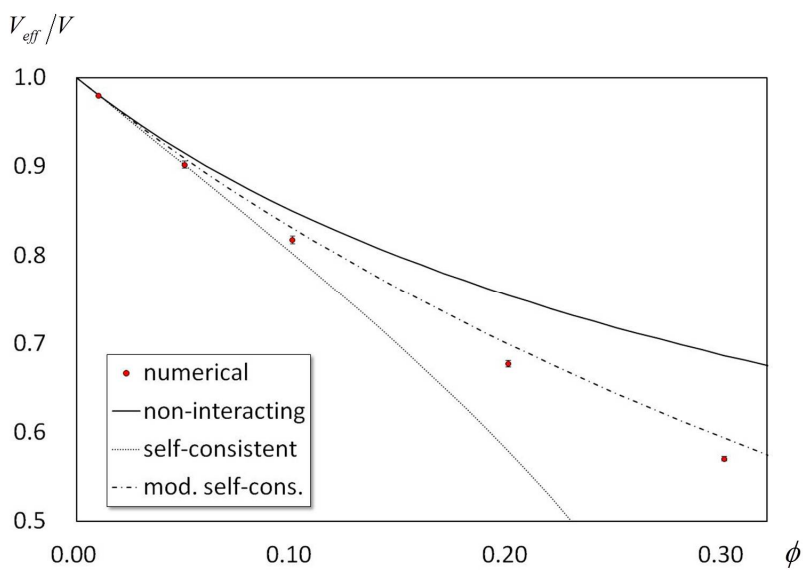

Figure 4 The calculated and theoretical effective velocities. Filled circles represent the calculated effective velocity. Solid, dotted and dashed lines represent the effective velocities from non-interacting, self consistent and modified self consistent theories, respectively. Vertical axis is normalized by back ground velocity $\mathrm{V}$.

FDM, FEM and $\mathrm{BEM}^{3), 9)}$.

\section{CONCLUSIONS}

We presented the application of HPM for simulating the seismic wave propagation and evaluating the effective velocity of P-wave in cracked media. HPM has some advantages to implement cracks inside the elastic media; i) it is easy to introduce free-surfaces, ii) the arbitrary spatial refinement is possible in a simple and easy manner.

In order to investigate the effectiveness of the 
method, we considered 2D isotropic cracked media. We predicted the effective P-wave velocity and compared them with those from three different theoretical approaches. Our results had good agreements with the effective velocities from modified self-consistent theory. This was consistent with the previous numerical studies conducted by FDM, FEM and BEM.

ACKNOWLEDGMENT: This study was partly supported by MEXT/JSPS KAKENHI Grant Number 24760361.

\section{REFERENCES}

1) Davis, P. M. and Knopoff, L., 1995, The elastic modulus of media containing strongly interacting antiplane cracks, J. Geophys. Res., 100, 18253-18258.

2) Henyey, F. S. and Pomphrey, N., 1982, Self-consistent elastic moduli of a cracked solid, Geophys. Res. Lett., 9, 903-906.

3) Saenger, E. H. and Shapiro, S. A., 2002, Effective velocities in fractured media: a numerical study using the rotated staggered finite-difference grid, Geophys. Prospect., 50, 183-194.
4) Saenger, E. H., Gold, N. and Shapiro, S. A., 2000, Modeling the propagation of elastic waves using a modified fnite-difference grid, Wave Motion, 31, 77-92.

5) Takekawa, J., Madariaga, R., Mikada, H. and Goto, T., in press, Numerical simulation of seismic wave propagation produced by earthquake by using a particle method, Geophys. J. Int., doi: 10.1111/j.1365-246X.2012.05676.x

6) Suzuki, Y. and Koshizuka, S., 2008, A Hamiltonian particle method for non-linear elastodynamics, Int. J. Num. Meth. Eng., 74, 1344-1373.

7) Takekawa, J., Madariaga, R., Mikada, H. and Goto, T., 2011, Applicability of a particle method with arbitrary particle refinement for elastic wave propagation, Proc. 10th SEGJ International Symposium - Imaging and Interpretation - , 290-293.

8) Kondo, M., Suzuki, Y. and Koshizuka, S., 2010, Suppressing local particle oscillations in the Hamiltonian particle method for elasticity, Int. J. Num. Meth. Eng., 81, 1514-1528.

9) Dahm, T. and Becker, TH., 1998, On the elastic and viscous properties of media containing strongly interacting in-plane cracks, Pure Appl. Geophys., 151, 1-16. 